\title{
Estudio neuroepidemiológico nacional (EPINEURO) colombiano
}

\author{
Gustavo Pradilla A., ${ }^{1}$ Boris E. Vesga A., ${ }^{2,3}$ \\ Fidias E. León-Sarmiento ${ }^{3}$ y grupo GENECO 4
}

RESUMEN

Objetivo. Determinar la prevalencia de ocho problemas neurológicos -migraña, enfermedad cerebrovascular, enfermedad de Parkinson, neuropatía periférica, trastornos del desarrollo neurológico, epilepsia, demencia y secuelas de traumatismo craneoencefálico- en Colombia como parte del estudio neuroepidemiológico nacional (EPINEURO).

Métodos. Se realizó una encuesta transversal en dos fases entre septiembre de 1995 y agosto de 1996. En la primera fase se identificó a las personas que podían estar padeciendo alguna enfermedad neurológica y en la segunda se hizo el diagnóstico específico. Se examinó una muestra de 8910 personas distribuidas en proporción con la población de cada una de las cinco zonas geográficas de Colombia, que son las regiones central, sudoccidental, noroccidental, oriental y la costa caribe. La evaluación se realizó según una versión modificada del protocolo de neuroepidemiología de la Organización Mundial de la Salud, además de un examen físico simplificado. A los posibles pacientes de demencia identificados se les aplicó un examen neuropsicológico, diseñado para este estudio, con el fin de profundizar en los aspectos cognoscitivos. Se aplicaron las escalas de Hachinski, Yesavage y Blessed y los criterios diagnósticos del NINCDS-ADRDA para la enfermedad de Alzheimer, los del NINDS-AIREN para la demencia de origen vascular y los del DSM-IV para todo tipo de demencia. Se realizó una encuesta para determinar si había secuelas de traumatismo craneoencefálico. Las personas mayores de 12 años fueron evaluadas por neurólogos de adultos y los menores de 12 años por un neuropediatra. Se calcularon las prevalencias de trastornos neurológicos por edad y sexo, tanto por región como para el país en su totalidad, y sus intervalos de confianza de 95\%.

Resultados. La prevalencia por 1000 habitantes de los trastornos investigados fueron las siguientes: migraña, 71,2 (intervalo de confianza de 95\% [IC95\%]: 65,5 a 76,8); trastornos del desarrollo neurológico, 46,1 (IC95\%: 35,5 a 58,9); enfermedad cerebrovascular, 19,9 (IC95\%: 14,3 a 27,4); demencia, 13,1 (IC95\%: 8,5 a 19,3); epilepsia, 10,3 (IC95\%: 8,5 a 13,0); neuropatía periférica, 8,5 (IC95\%: 6,8 a 10,7); secuelas de traumatismo craneoencefálico, 6,4 (IC95\%: 5,0 a 7,8) y enfermedad de Parkinson 4,7 (IC95\%: 2,2 a 8,9). En todos los casos, menos en el de traumatismos craneoencefálicos, la prevalencia fue mayor en mujeres.

Conclusiones. Estos resultados permiten diseñar políticas de salud más actuales dirigidas a lograr una prevención más eficaz, un mejor control y un tratamiento más adecuado de los trastornos neurológicos en Colombia. La fácil aplicación de las encuestas y el bajo costo de este tipo de investigaciones hacen que esta metodología sea ideal para países con carencia de recursos humanos especializados.

Palabras clave Migraña, trastornos cerebrovasculares, enfermedad de Parkinson, polineuropatía, epilepsia, demencia, trauma craneocerebral, epidemiología, Colombia.

1 Universidad Industrial de Santander, Bucaramanga, Colombia.

2 Universidad Autónoma de Bucaramanga Bucaramanga, Colombia.

3 Universidad Industrial de Santander, Facultad de Salud, Departamentos de Medicina Interna y
Ciencias Básicas, Bucaramanga, Colombia. Dirigir la correspondencia a: Fidias E. León-Sarmiento, Medical Neurology Branch, National Institutes of Health, 10 Center Drive, Building 10, Room 5N 234, Bethesda MD 20892, Estados Unidos de América. Correo electrónico: leonf@ninds.nih.gov.
4 Grupo GENECO: Diego A. Roselli, Leonelo E. Bautista, Luis Morillo, Carlos S. Uribe, Yuri Takeuchi, Jorge Daza, Eugenia Espinoza, Manuel I. Camacho y Carlos A. Pardo. 
Los trastornos neurológicos en su conjunto constituyen una de las principales causas de muerte y discapacidad en el mundo y afectan a todos los grupos de edad. No obstante, se cuenta con muy poca información acerca de su frecuencia y distribución en los países en desarrollo. Los principales datos en este sentido provienen de los registros de egreso de instituciones de atención médica, los cuales son por lo general deficientes debido a que muchas personas con trastornos neurológicos nunca solicitan atención médica, se automedican o buscan la ayuda de personas que no son profesionales (1). Estos hechos, entre muchos otros, son los que generan un subregistro de casos (2) y los que hacen que sea necesario recurrir a otras estrategias para cuantificar de forma válida y precisa la prevalencia de estos trastornos en la población. Entre estas estrategias se encuentra la realización de encuestas epidemiológicas transversales de muestras grandes de población (3).

En Colombia, este tipo de investigación es aún escaso y solo se destacan siete estudios sobre la prevalencia de enfermedades neurológicas realizados entre 1983 y 1992 (4) con metodologías similares, basadas en el protocolo para enfermedades neurológicas diseñado por la Organización Mundial de la Salud (OMS) (5). Sin embargo, esos estudios tuvieron algunas limitaciones. Por ejemplo, la mayoría de ellos se llevaron a cabo con muestras pequeñas de población, lo que originó resultados estadísticamente imprecisos; aunque todos se basaron en el protocolo de la OMS, no hubo homogeneidad en la identificación de casos; y algunos de los estudios se hicieron en poblaciones expuestas a sustancias tóxicas (6-8) o con una alta prevalencia de alguna enfermedad neurológica, como la corea de Huntington (9), lo que limitó la posibilidad de extrapolar sus resultados a la población en general.

Por todo lo antedicho, se decidió realizar simultáneamente en las cinco regiones geográficas de Colombia este estudio neuroepidemiológico nacional, denominado EPINEURO, con el objetivo principal de determinar las prevalencias de epilepsia, migraña, enfermedad cerebrovascular (ECV), enfermedad de Parkinson, neuropatía periférica y trastornos del desarrollo neurológico. Se determinaron además las prevalencias de demencia y de secuelas de traumatismo craneoencefálico, dos trastornos neurológicos de gran importancia en la actualidad acerca de los cuales no se tenía información en Colombia. Los estudios por regiones han sido publicados por separado (10-13). En este trabajo se presentan los resultados consolidados del estudio EPINEURO.

\section{MATERIALES Y MÉTODOS}

Entre septiembre de 1995 y agosto de 1996 se realizó una encuesta transversal en dos fases. En la primera, se identificó a individuos que pudieran estar padeciendo algún trastorno neurológico y en la segunda se realizó el diagnóstico específico del mismo. Esta estrategia ha sido recomendada por la OMS para estudios poblacionales de enfermedades neurológicas en países en desarrollo, ya que permite optimar los recursos humanos disponibles (5).

Durante la primera fase, la de tamizaje, el equipo de encuestadores realizó visitas de puerta en puerta a las viviendas escogidas de manera aleatoria para formar la muestra del estudio. Las personas seleccionadas fueron evaluadas según una versión modificada del protocolo de neuroepidemiología de la OMS (10-13) y a todas se les realizó el examen neurológico contemplado en él. Con el fin de identificar posibles casos de demencia, a los mayores de 50 años se les aplicó una versión del examen mental abreviado (minimental), traducida al español y ajustada a las circunstancias locales (14). Cuando los resultados del examen mental abreviado realizado durante el tamizaje indicaron que la persona podría estar padeciendo de demencia, se aplicó un examen neuropsicológico diseñado para este estudio con el fin de profundizar en los aspectos cognitivos analizados mediante la prueba inicial. Se aplicaron las escalas de Hachinski (15), Yesavage (16) y Blessed (17) y la clasificación de demencia clínica (18). Para la enfermedad de Alzheimer se utilizaron los criterios diagnósticos NINCDSADRDA (elaborados conjuntamente por el National Institute of Neurological and Communicative Disorders and Stroke y la Alzheimer's Disease and Related Disorders Association) (19) y para la demencia vascular, los NINDS-AIREN (elaborados por el National Institute for Neurological Disorders and Stroke conjuntamente con la Association Internationale pour la Recherche et l'Enseignement en Neurosciences) (20). Los criterios de la cuarta edición del Manual para el diagnóstico y las estadísticas de enfermedades mentales (DSM-IV) fueron empleados para evaluar los casos de demencia (21). Por último, durante el tamizaje se realizó una entrevista dirigida a identificar a las personas que habían sufrido algún traumatismo craneoencefálico grave que pudiera haber dejado secuelas neurológicas; en este caso, el criterio de inclusión fue que el paciente hubiera perdido el conocimiento o que hubiera sido necesario someterlo a un procedimiento neuroquirúrgico (10-13). Las personas con signos de haber estado padeciendo alguna enfermedad neurológica durante el tamizaje fueron referidas a una segunda fase, en la que un neurólogo o un neuropediatra (para los menores de 12 años) evaluó cada caso. Estos especialistas no tenían conocimiento de los hallazgos del tamizaje y utilizaron guías y criterios específicos predefinidos para el diagnóstico de las enfermedades neurológicas (1-14).

Fueron elegibles para participar en este estudio todas las personas residentes en Colombia durante el período de estudio (1995-1996). Siguiendo las definiciones del Departamento Administrativo Nacional de Estadística (DANE) (22), se escogieron cinco ciudades en las que se ubicaron los centros operativos regionales del estudio de la siguiente manera: Bogotá, en la región central; Cali, en la sudoccidental; Medellín, en la noroccidental; Bucaramanga, en la oriental; y Barranquilla, en la costa caribe. Las ciudades que operarían como centros regionales del estudio se seleccionaron según la disponibilidad de neurólogos y por la existencia en ellas de una facultad de medicina que 
CUADRO 1. Cálculo del tamaño de la muestra, precisión y número de casos esperados en el estudio neuroepidemiológico nacional (EPINEURO), Colombia

\begin{tabular}{|c|c|c|c|c|c|}
\hline Enfermedad & Prevalencia $^{a}$ & Precisión & Casos & Precisión ${ }^{b}$ & No. ${ }^{c}$ \\
\hline Epilepsia & 0,0160 & 0,40 & 3779 & 0,31 & 104 \\
\hline Trastornos del desarrollo neurológico & 0,0073 & 0,20 & 6955 & 0,21 & 47 \\
\hline Enfermedad cerebrovascular & 0,0065 & 0,20 & 6199 & 0,20 & 42 \\
\hline Enfermedad de Parkinson & 0,0057 & 0,18 & 6716 & 0,18 & 37 \\
\hline \multicolumn{6}{|l|}{ Secuelas de traumatismo } \\
\hline
\end{tabular}

a Prevalencia obtenida de estudios anteriores, a partir de la cual se calculó la muestra (ver métodos)

b Precisión del estudio si se tomara una muestra de 6500 personas.

c Número de casos que produciría el estudio con una muestra de 6500 personas.

¿ En el caso de las demencias se utilizó una $n=3000$.

pudiera apoyar el estudio. Debido a que la prevalencia de trastornos neurológicos y, sobre todo, de secuelas de traumatismo craneoencefálico podían variar de acuerdo con el nivel de urbanización de la población, en cada región se eligió una zona urbana, una semiurbana y una rural. Como en las zonas rurales no había conglomerados de viviendas con un determinado número de habitantes que se correspondieran con el concepto de barrio, se hizo un levantamiento cartográfico en esas zonas y se seleccionó la muestra de la misma forma que se hizo en los barrios de la zonas urbanas y semiurbanas (10).

Los valores de prevalencia usados para calcular el tamaño de la muestra fueron tomados de los estudios realizados previamente en Colombia. En el caso de los trastornos cuya frecuencia no había sido definida en la población colombiana, como las secuelas de traumatismo craneoencefálico, se usaron valores tomados de la bibliografía médica en general (23). Se calculó el tamaño muestral mínimo para cada uno de los trastornos neurológicos que se deseaba estudiar y se escogió el mayor de todos los tamaños. En vista de que los trastornos neurológicos son más frecuentes a medida que aumenta la edad, se aumentó el tamaño de la muestra de personas mayores de 50 años con el objetivo de mejorar la productividad del estudio. Esto compensaría la cantidad de personas que pudieran negarse a participar y las pérdidas que pudieran ocurrir entre la primera y la segunda fase del estudio. Además, una mayor participación de personas mayores de 50 años permitiría obtener más casos de trastornos neurológicos y, por lo tanto, valores de prevalencia más precisos. En consecuencia, el tamaño de muestra planificado fue de 8720 personas. La distribución de la muestra se hizo de manera proporcional según la población de cada una de las regiones del país (cuadro 1).

A los residentes de las poblaciones seleccionadas se les explicó la importancia del estudio y de su participación en él. En esta tarea participaron promotores rurales, juntas de acción comunal, maestros de escuela, líderes cívicos y, donde fue posible, trabajadores sociales. El grupo de trabajo estuvo integrado por neurólogos, neuropediatras y epidemiólogos. Además, participaron médicos generales y estudiantes de ciencias de la salud, quienes se encargaron de realizar el examen físico simplificado y las encuestas de la fase de tamizaje, respectivamente. Estos fueron entrenados por los neuró$\log$ os con el fin de que estuvieran debidamente calificados y se garantizara la homogeneidad de los datos recolectados en formatos precodificados y estandarizados.

Teniendo en cuenta que a la segunda fase pasaban aquellas personas que durante el tamizaje mostraron signos de padecer alguna enfermedad neurológica, la prevalencia podría resultar sobrestimada. Para reducir este sesgo, se seleccionó para la segunda fase una muestra aleatoria equivalente a $5 \%$ de las personas que no presentaron indicios de enfermedad neurológica.

Una vez finalizado el estudio de un participante, todas sus encuestas eran revisadas por el coordinador regional de la investigación, quien discutía de inmediato con los encuestadores o el neurólogo las incoherencias detectadas y las corregía. Luego, los datos se introdujeron por duplicado en bases de datos (en formato dBase) y se reevaluaron y corrigieron los errores de coherencia y tecleado. Las prevalencias de trastornos neurológicos expresadas por 1000 habitantes se calcularon por edad y sexo, tanto por región como para el país en su totalidad. Para esto se dividió el número de casos idenidentificados entre el tamaño de la población encuestada en la que fueron detectados los pacientes. Como durante el muestreo se favoreció la participación de personas mayores de 50 años, se hizo un ajuste directo de las 
CUADRO 2. Número de casos y prevalencia de trastornos neurológicos según el estudio neuroepidemiológico nacional (EPINEURO), Colombia

\begin{tabular}{|c|c|c|c|c|}
\hline \multirow[b]{2}{*}{ Trastorno } & \multicolumn{3}{|c|}{ Número de casos } & \multirow[b]{2}{*}{ Prevalencia $^{\mathrm{C}}$} \\
\hline & Total & Hombres & Mujeres & \\
\hline Migraña & 634 & 144 & 490 & 71,2 (IC95\%: 65,5 a 76,8) \\
\hline Trastornos del desarrollo neurológico ${ }^{a}$ & 63 & 38 & 25 & 46,1 (IC95\%: 35,5 a 58,9 ) \\
\hline Enfermedad cerebrovascular ${ }^{\mathrm{b}}$ & 38 & 16 & 22 & 19,9 (IC95\%: 14,3 a 27,4) \\
\hline Demenciab $^{b}$ & 25 & 7 & 18 & 13,1 (IC95\%: 8,5 a 19,3$)$ \\
\hline Epilepsia & 92 & 31 & 61 & 10,3 (IC95\%: 8,5 a 13,0) \\
\hline Neuropatía periférica & 76 & 30 & 46 & 8,5 (IC95\%: 6,8 a 10,7) \\
\hline Secuelas de traumatismo craneoencefálico & 57 & 27 & 30 & 6,4 (IC95\%: 5,0 a 7,8) \\
\hline Enfermedad de Parkinson ${ }^{c}$ & 9 & 7 & 2 & 4,7 (IC95\%: 2,2 a 8,9) \\
\hline
\end{tabular}

IC95\%: intervalo de confianza de $95 \%$

a Evaluada en menores de siete años.

b Evaluada en mayores de 50 años.

${ }^{c}$ Prevalencia por 1000 habitantes, ajustada por edad y sexo.

prevalencias con el fin de obtener valores que reflejaran la situación real de la población estudiada. Este ajuste se realizó usando como factores de ponderación los porcentajes dados por la distribución por edades de las regiones estudiadas, según los datos del censo nacional de 1993 (22). Además, se calcularon intervalos de confianza de $95 \%$ para las prevalencias ajustadas por considerarse que estas tenían una distribución normal (24).

\section{RESULTADOS}

Se entrevistó a 8910 personas residentes de 2560 viviendas (1 303 personas en la región oriental; 3077 en la central; 1591 en la noroccidental; 998 en la sudoccidental; y 1941 en la costa caribe). La proporción de mujeres fue de $60 \%$, cifra mayor que la esperada $(51,5 \%)$ según los datos del censo nacional de 1993 (22). Por otra parte, el promedio de edad de las personas que participaron en el estudio fue mayor que el de la población de la cual se tomó la muestra. Esta diferencia se debió a que, con el fin de aumentar el número de casos de enfermedades neurológicas y obtener valores de prevalencia más precisos, se aumentó el número de personas mayores de 50 años en la muestra. Consecuentemente, cuando fue necesario se ajustaron las prevalencias totales de los distintos trastornos neurológicos estudiados, según la distribución de los grupos de edad de cada población (cuadro 2).

En cuanto a las enfermedades estudiadas, $77,3 \%$ de los casos de migraña fueron en mujeres. En general, la prevalencia fue aproximadamente dos veces mayor en mujeres que en hombres; mayor en la región oriental y menor en la costa caribe. Esta enfermedad fue poco frecuente en la población infantil (solo 3,5\% tenían menos de 10 años de edad) y se observó un incremento gradual de la prevalencia hasta los 19 años de edad. A partir de la tercera década de la vida, la prevalencia de migraña continuó aumentando, aunque en menor proporción, y experimentó un descenso progresivo en personas mayores de 50 años. Estos cambios en la prevalencia fueron muy similares en hombres y mujeres, aunque en los primeros las tendencias no estaban bien definidas debido al reducido número de casos.

La prevalencia de epilepsia fue mayor en mujeres que en hombres $(13,8$ frente a 10,1 por 1000 habitantes). No se encontró un patrón definido de cambios con la edad, aunque se observó un incremento en el grupo de 20 a 29 años. La prevalencia fue mayor en las regiones oriental y noroccidental. Con respecto a la neuropatía periférica, esta fue $30 \%$ mayor en mujeres que en hombres, principalmente en mayores de 40 años, y predominó la mononeuropatía
(65,8\% de los casos de neuropatía). Las regiones más afectadas fueron la oriental y la sudoccidental. La ECV presentó una mayor prevalencia en personas de más de 50 años, con valores superiores en las mujeres. Las regiones con mayor prevalencia fueron la sudoccidental y la noroccidental.

La enfermedad de Parkinson fue detectada en personas mayores de 60 años, y más de la mitad de los pacientes vivían en la región noroccidental. Por su parte, la prevalencia de secuelas de traumatismo craneoencefálico fue mayor entre los hombres (7,5 por 1000 habitantes), especialmente entre los de más de 65 años $(23,6$ por 1000 habitantes). Las mayores frecuencias se observaron en la costa caribe y en la región noroccidental. Con respecto a los trastornos del desarrollo neurológico, se encontró que hasta $39,7 \%$ de los casos fueron de características mixtas, con alteraciones motoras, cognoscitivas y del lenguaje. La mayor prevalencia se encontró en la región oriental $(66,3$ por 1000 habitantes). Finalmente, la demencia se detectó solo en personas mayores de 50 años de edad y su prevalencia fue superior en los mayores de 70 años $(30,4$ por 1000 habitantes). Las regiones más afectadas fueron la sudoccidental y la oriental con 21,9 y 19,3 por 1000 habitantes, respectivamente. 


\section{DISCUSIÓN}

Este es el primer estudio de prevalencia de estos trastornos neurológicos en una muestra representativa de toda la población colombiana. Aunque el promedio de edad de la muestra estudiada era mayor que el de la población en general, el uso de prevalencias ajustadas por edad permitió corregir este error y obtener valores representativos de la población de todo el país. Por otra parte, el predominio de mujeres en la población encuestada puede deberse a que las mujeres son más receptivas a participar en encuestas de este tipo y a que en el horario en que se realizaron estas encuestas eran más los hombres que se encontraban fuera de casa por razones laborales.

La migraña es una enfermedad que, aunque no es grave, tiene un alto impacto sobre el bienestar y la capacidad laboral de las personas afectadas (25). Sin embargo, en Colombia no existía una adecuada información sobre su frecuencia (26). Las prevalencias de $1 \%$ a $25 \%$ encontradas en otros países, entre ellos algunos donde se empleó este mismo protocolo (26-32), son comparables a la de 71,2 por 1000 habitantes hallada en este estudio. Este valor, sin embargo, es menor que el informado anteriormente para la población colombiana, lo que puede deberse a que en esta ocasión se abarcaron regiones geográficas y estratos de población no estudiados anteriormente. También se debe tener en cuenta que en los otros estudios no se estandarizó la estrategia de identificación de casos, las muestras empleadas fueron relativamente pequeñas y los resultados no fueron ajustados según la distribución de edades de la población colombiana. La mayor prevalencia hallada entre las mujeres coincide con lo publicado por otros autores, lo mismo que el aumento observado hasta la tercera década de la vida y su disminución relativa en los grupos de mayor edad $(33,34)$. Por otra parte, las diferencias entre las prevalencias de las cinco regiones colombianas podrían estar asociadas con factores de tipo genético, familiar y cultural. Futuros análisis de los datos del estudio EPINEURO podrían profundizar más en las causas de estas diferencias.

La expresión "trastorno del desarrollo neurológico" es vaga y en ocasiones se prefiere utilizar en su lugar el término "retardo psicomotor" (10-13). En la presente investigación, dentro de los trastornos del desarrollo neurológico se agruparon los casos de retardo del desarrollo cognoscitivo, del desarrollo del lenguaje y del desarrollo motor. La prevalencia encontrada en menores de 7 años fue aproximadamente el doble de la hallada en otros países (35). Esta diferencia podría ser el resultado de la adición de diversos factores, como los riesgos prenatal, perinatal y postnatal, así como de determinadas características socioculturales. Sin embargo, también es posible que se deba al uso de metodologías diferentes, especialmente por la inclusión dentro de esta categoría de los casos con trastornos en el desarrollo del lenguaje. Por estas razones no es posible comparar los resultados de los estudios pilotos colombianos previos con los del estudio EPINEURO en cuanto a los trastornos del desarrollo neurológico. Sin embargo, la elevada prevalencia hallada amerita un análisis posterior, especialmente si se tiene en cuenta que se trata de afecciones que pueden durar toda la vida y tener un alto costo humano, financiero y social.

La demencia es un problema clínico que tiene numerosas causas, algunas conocidas y otras no. De ellas, la enfermedad de Alzheimer es la más frecuente $(36,37)$. Estudios poblacionales sobre esta enfermedad realizados recientemente en España han revelado prevalencias de 13 a $15 \%$ en personas mayores de 65 años, con predominio en mujeres e incrementos con la edad $(38,39)$. En un estudio en la población francesa, en el que también se empleó el protocolo de la OMS, se encontró una prevalencia de demencia de $0,37 \%$ en personas mayores de 40 años (40), similar a la encontrada en este estudio en mayores de 50 años. El predominio de casos en la población femenina podría estar asociado también a la mayor longevidad de las mujeres.
En cuanto a la epilepsia, existen estudios de prevalencia en más de 25 países de diversas regiones del mundo con valores entre 1,5 y 57 por 1000 habitantes (41), por debajo de 10 por 1000 habitantes en países desarrollados y mucho más elevados en países en vías de desarrollo. Por ejemplo, un estudio realizado en Sicilia mediante una metodología similar a la utilizada en este trabajo reveló una prevalencia de epilepsia activa en 2,7 por 1000 habitantes (42), mientras que la encontrada en Colombia es de 10,3 por 1000 habitantes. Debido a la rigurosidad metodológica de este trabajo y al ajuste por edad realizado, los presentes resultados deben reflejar la verdadera prevalencia de esta enfermedad en Colombia y podrían indicar una disminución de la prevalencia de epilepsia en los últimos años (43). Un estudio hecho en Inglaterra mostró que la epilepsia activa se redujo de 5,3 por 1000 habitantes en 1983 a 4,3 por 1000 habitantes en 1993. Aunque pequeña, la reducción observada en esta población hace plausible que cambios similares puedan ocurrir en otros países como Colombia, si se toman las medidas preventivas indicadas para esta enfermedad. De hecho, hay indicios de que la incidencia de epilepsia en niños está disminuyendo $(43,44)$.

La falta de definiciones estandarizadas de neuropatía periférica y la heterogeneidad de sus causas, sus mecanismos patológicos y su gravedad (45) hacen que sea difícil describir los patrones poblacionales de la ocurrencia de esta enfermedad. Como consecuencia, la información acerca del comportamiento epidemiológico de este trastorno neurológico es escasa. En una encuesta puerta a puerta realizada en la India se encontró una prevalencia de neuropatía periférica de $2,4 \%$ y los diagnóstico más comunes fueron el síndrome del túnel del carpo y la neuropatía periférica diabética (46). Por otra parte, en un estudio hecho en Italia, $8 \%$ de las personas mayores de 55 años que consultaron al médico tuvieron alguna manifestación de polineuropatía, y la diabetes fue la enfermedad asociada 
más frecuente (47). En EPINEURO predominó la mononeuropatía, con una mayor prevalencia en mujeres mayores de 40 años, aunque hasta el momento no se conocen estudios que indiquen que el género sea un factor de riesgo.

La prevalencia de secuelas de traumatismo craneoencefálico encontrada en este estudio es superior a la publicada para Canadá, Estados Unidos de América y Escocia (48), lo cual podría estar asociado con los problemas socioculturales que aquejan a Colombia, en especial los derivados de la violencia. Su mayor frecuencia en hombres puede explicarse por su mayor vinculación con hechos violentos, aunque llama la atención la alta prevalencia en mayores de 65 años. Estos hallazgos confirman la necesidad de lograr una mayor precisión de los posibles factores de riesgo para poder desarrollar medidas preventivas eficaces. Además, se deben proveer los cuidados sanitarios apropiados para la atención y rehabilitación de estos pacientes.

Con respecto a la ECV, su prevalencia se redujo a la mitad de la observada en estudios realizados en Colombia con anterioridad $(1,4,6$, $7-14,49)$ y fue menor que las observadas en países industrializados. Las prevalencias se encuentran entre 5 por 1000 habitantes en Estados Unidos y Europa y 9 por 1000 habitantes en países de Asia Oriental (50). El hecho de que durante las últimas décadas la mortalidad por ECV no haya experimentado cambios notables favorece la hipótesis de que la contradicción observada entre las prevalencias de ECV en Colombia según los diferentes estudios se debe a efectos del muestreo, sobre todo teniendo en cuenta el pequeño tamaño de muestra de los estudios anteriores. Dada la mejoría en la atención de pacientes con ECV durante los últimos años, sería natural esperar un aumento de la esperanza de vida de estos casos $\mathrm{y}$, en consecuencia, un aumento de la prevalencia. Sin embargo, este aumento puede haber sido anulado o revertido por la disminución en la incidencia de ECV como resultado de una adecuada detección y un acertado tratamiento de la hipertensión arterial - uno de los principales factores de riesgo de la ECV - o por el control de otros factores, como la neurotripanosomiasis americana. Se requieren investigaciones adicionales que aborden este tema (51-53).

Los estudios epidemiológicos de la enfermedad de Parkinson presentan diversas limitaciones debido al reducido número de casos, incluso en estudios de grandes poblaciones (14). La prevalencia hallada en la presente investigación fue nueve veces mayor que la encontrada en Colombia con anterioridad, muy similar a la descrita en Taiwán y casi la mitad de la observada en algunos países europeos (54). El cálculo de las prevalencias ajustadas por edad para 27 poblaciones y 45 comunidades permitió a Zhang y colaboradores (54) establecer que -con excepción de China, Japón y la Región de África, cuyas prevalencias son más bajas - las variaciones son probablemente menores que las informadas con anterioridad para poblaciones geográficamente diversas. Además, estos autores señalan que es poco probable que las variaciones en la prevalencia de la enfermedad de Parkinson observadas entre diferentes regiones se deban exclusivamente a factores raciales, ya que los factores de riesgo ambientales pueden influir en el número de casos de esta enfermedad a escala regional.

En conclusión, estos resultados ofrecen una visión mucho más clara de la situación epidemiológica de los trastornos neurológicos en Colombia y podrían servir de base para el establecimiento de políticas de salud modernas, orientadas hacia una prevención más eficaz, un mejor control y un tratamiento más adecuado de estas afecciones neurológicas en Colombia. La consideración de estos resultados durante la elaboración de los programas académicos de ciencias de la salud, tanto de pregrado como de posgrado, es otro de los beneficios derivados del presente trabajo. La fácil aplicación de las encuestas y el bajo costo de este tipo de investigaciones hacen que esta metodología sea ideal para países con carencia de recursos humanos especializados.

Agradecimiento. Esta investigación fue financiada por la Dirección de Desarrollo Científico y Tecnológico del Ministerio de Salud de Colombia y la Dirección de Investigaciones de la Facultad de Salud de la Universidad Industrial de Santander (Bucaramanga). Los autores agradecen la colaboración prestada por los estudiantes de medicina y ciencias de la salud de la Universidad Industrial de Santander, la Pontificia Universidad Javeriana (Bogotá), la Universidad de Antioquia (Medellín), la Universidad del Valle (Cali) y la Universidad del Norte (Barranquilla) durante la realización de esta investigación.

\section{REFERENCIAS}

1. Pradilla G, Puentes F, Pardo C. Estudio neuroepidemiológico piloto. Acta Neurol Colomb 1984;8:133-139.

2. Alfonso H, León-Sarmiento FE, Brokate AM, Valderrama V, Parra JE. Epidemiología del SIDA basada en la evidencia. En: LeónSarmiento FE, ed. VIH y los virus de la ima- ginación humana. Santa Fe de Bogotá: Celsus; 2001. Pp. 13-32.

3. Schoenberg BS. Clinical neuroepidemiology in developing countries. Neuroepidemiology 1982;1:136-142.

4. Pradilla G, Pardo CA, Zúñiga A, Daza JS. Estudios neuroepidemiológicos colombianos empleando el protocolo de la OMS. Rev Neurol Arg 1994;19:9-14.

5. World Health Organization. Research protocol for measuring the prevalence of neurological disorders in developing countries. Geneva: World Health Organization, Neurosciences Program; 1981. 
6. León-Sarmiento FE, Pradilla G, Pardo CA, Puentes F. The first neuroepidemiological study performed in Colombia using the World Health Organization protocol. Neurosciences (Tokyo) 1993;19:127-130.

7. Pradilla G, Vesga E, Sánchez LH. Estudio neuroepidemiológico y toxicológico de una población minera con exposición crónica al mercurio. Acta Med Colomb 1992;17: 322.

8. Pradilla G, Vesga E, Sánchez LH. Estudio neuroepidemiológico y neurotoxicológico de plaguicidas en una zona algodonera del Sur del César, Colombia. Acta Med Colomb 1992;17:324.

9. Daza B, Caiaffa RH, Arteta BJ. Estudio neuroepidemiológico en Juan de Acosta, Atlántico, Colombia. Acta Med Colomb 1992;17:324.

10. Pradilla G, Vesga BE, León-Sarmiento FE. Estudio neuroepidemiológico en Aratoca: un área rural del oriente colombiano. Rev Med Chile 2002;130:191-199.

11. Pradilla G, Vesga BE, León-Sarmiento FE, Bautista LE, Núñez LC, Vesga E, et al. Neuroepidemiología en el oriente colombiano. Rev Neurol 2002;34:1035-1043.

12. Takeuchi Y, Guevara JG. Prevalencia de las enfermedades neurológicas en el Valle del Cauca. Colomb Med 1999;30:74-81.

13. Pradilla G, Roselli D, Bautista L, Morillo L, Uribe CS, Takeuchi Y, et al. Neuroepidemiología en Colombia. Acta Med Colomb 1998; 23:258.

14. León-Sarmiento FE, Pradilla G, Villamil L, Vesga BE, Dallos M. Análisis crítico del minimental: 1975-1995. Acta Neurol Colomb 1999; 15:135-145.

15. Chui HC, Victoroff JI, Margolin D. Criteria for the diagnosis of ischemic vascular dementia proposed by the state of California Alzheimer's Disease Diagnosis and Treatment Centers. Neurology 1992;42:473-480.

16. Yesavage JA. Geriatric depression scale. Psychopharmacol Bull 1988;24:709-711.

17. Blessed G, Tomlinson BE, Roth M. Blessedroth dementia scale. Psychopharmacol Bull 1988;24:705-708.

18. Berg L. Clinical Dementia Rating (CDR). Psychopharmacology Bull 1988;24:637-639.

19. McKhann G, Drachman D, Folstein M, Katzman R, Price D, Stadlan EM. Clinical diagnosis of Alzheimer's disease: report of the NINCDS-ADRA Work Group under the auspices of Department of Health and Human Services Task Force on Alzheimer's Disease. Neurology 1984;34:939-944.

20. Román GC, Tatemichi TK, Erkinjuntti T, Cummings JL, Masdeu JC, García JH. Vascular dementia: diagnostic criteria for research studies. Report of the NINDSAIREN International Workshop. Neurology 1993;43:250-260.

21. American Psychiatric Association. Diagnostic and statistical manual of mental disorders. 4th edition. Washington, D.C.: American Psychiatric Association; 1994.

22. Departamento Administrativo Nacional de Estadística. Censo de población y vivienda 1993. Proyecciones de población para 1995. Santa Fe de Bogotá: DANE; 1995.
23. Kraus JF, McArthur DL. Epidemiological aspects of brain injury. Neurol Clin 1996;14: 435-450.

24. Esteve J, Benhamou E, Raymond L. Descriptive epidemiology. Lyon: IARC Scientific Publications; 1994. (IARC Scientific Publications No. 128).

25. León-Sarmiento FE, Martínez M, García I. Migraña y calidad de vida en trabajadores hospitalarios colombianos, usando los protocolos de la OMS y MIDAS (MIDASELA), en español. Rev Neurol 2003;36:412-417.

26. Pradilla G, León-Sarmiento FE. Migraine prevalence. Neurology 1995;45:1030.

27. Silberstein SD, Lipton RB. Headache epidemiology: emphasis on migraine. Neurol Clin 1996;14:421-434.

28. Cruz ME. Estudio neuroepidemiológico en el Ecuador. Quito: CIEN, Fundación Eugenio Espejo, Ministerio de Salud Pública; 1984.

29. Osuntokun BO, Schoenberg BS, Nottidge V, Adeuja A, Kale O, Adeyefa A, et al. Migraine headache in a rural community in Nigeria: results of a pilot study. Neuroepidemiology 1982;1:31-39.

30. Ponce PL. Estudios neuroepidemiológicos en Venezuela. Caracas: Departamento de Enfermedades Neurológicas, Ministerio de Sanidad y Asistencia Social; 1985.

31. Cruz Gutiérrez del Olmo A, Schoenberg BS, Portera-Sánchez A. Prevalence of neurological diseases in Madrid, Spain. Neuroepidemiology 1989;8:43-47.

32. Zhao F, Tasy JY, Cheng X, Wong W, Li S, Yao $S$, et al. Epidemiology of migraine: a survey in 21 provinces of the People's Republic of China, 1985. Headache 1988;28:558-565.

33. Stewart WF, Shechter A, Rasmussen BK. Migraine prevalence: a review of populationbased studies. Neurology 1994;44(Suppl 4): 17-23.

34. Ziegler DK. Epidemiology of migraine. En: Anderson DW, ed. Neuroepidemiology: a tribute to Bruce Schoenberg. Boca Raton: CRC Press; 1991. Pp. 167-192.

35. Stein Z, Durkin M. Serious mental retardation in developing countries: an epidemiologic approach. Ann NY Acad Sci 1986;477:8-21.

36. León-Sarmiento FE, de Castro Costa CM, Gaffga N. Discrepancy, coincidence or evidence in chronic idiopathic spastic paraparesis throughout the world. Arq Neuropsiquiat 1997;55(3B):530-535.

37. Rocca WA, Amaducci L. Epidemiology of Alzheimer's disease. In: Anderson DW, ed. Neuroepidemiology: a tribute to Bruce Schoenberg. Boca Raton: CRC Press; 1991. Pp. 55-96.

38. López S, Llinás J, Vilalta J, Lozano L. The prevalence of dementia in Girona. Neurología 1995;10:189-193.

39. Pi J, Olivé JM, Roca J, Masana L. Prevalence of dementia in a semi-rural population of Catalunya, Spain. Neuroepidemiology 1996; 15:33-41.

40. Munoz M, Boutros-Toni F, Preux PM, Chartier JP, Ndzanga E, Boa F. Prevalence of neurological disorders in Haute-Vienne department (Limousin region-France). Neuroepidemiology 1995;14:193-198.
41. Sanders JWAS, Shorvon SD. Epidemiology of the epilepsies. J Neurol Neurosurg Psychiatry 1996;61:433-443.

42. Reggio A, Failla G, Patti F, Nicoletti A, Grigoletto F, Meneghini F. Prevalence of epilepsy. A door-to-door survey in the Sicilian community of Riposto. Ital J Neurol Sci 1996;17:147- 151

43. Vesga BE. Neuroepidemiología de la epilepsia. Salud UIS 2001;33:229-235.

44. Cockerell OC, Eckle I, Goodridge DM, Sander JW, Shorvon SD. Epilepsy in a population of 6.000 re-examined: secular trends in first attendance rates, prevalence, and prognosis. J Neurol Neurosurg Psychiatry 1995;58:570576.

45. Pradilla G, León-Sarmiento FE, Gamarra G. Síndrome de Guillain-Barre en el oriente colombiano. Acta Neurol Colomb 1999;15:1423.

46. Bharucha NE, Bharucha AE, Bharucha EP. Prevalence of peripheral neuropathy in the Parsi community of Bombay. Neurology 1991; 41:1315-1317.

47. Beghi E, Monicelli ML, Amoruso L, Apollo F, Delodovivi ML, Grampa G. Chronic symmetric symptomatic polyneuropathy in the elderly: a field screening investigation in two Italian regions. Prevalence and general characteristics of the sample. Neurology 1995;45: 1832-1836.

48. Jennett B. Epidemiology of head injury. J Neurol Neurosurg Psychiat 1996;60:362-369.

49. Uribe CS, Jiménez I, Mora MO, Arana A Sánchez JL, Zuluaga L. Epidemiología de las enfermedades cerebrovasculares en Sabaneta, Colombia (1992-1993). Rev Neurol 1997;25: 1008-1012.

50. Davis PH, Hachinski V. Epidemiology of cerebrovascular disease. In: Anderson DW, ed. Neuroepidemiology: a tribute to Bruce Schoenberg. Boca Raton: CRC Press; 1991. Pp. 27-53.

51. León-Sarmiento FE, Mendoza E, Prada J, Silva CA, Vera SJ, Castillo E. Enfermedad cerebrovascular y Trypanosoma cruzi en el oriente colombiano. Acta Neurol Colomb 2001;17: 171-177.

52. León-Sarmiento FE, Mendoza E, TorresHillera M. Trypanosoma cruzi-associated cerebrovascular disease: A case-control study at Eastern Colombia. J Neurol Sci (en prensa).

53. Pinto NX, Torres-Hillera M, Mendoza $\mathrm{E}_{\text {, }}$ León-Sarmiento FE. Immune response, nitric oxide, autonomic dysfunction and stroke: a puzzling linkage on $T$. cruzi infection. Med Hypoth 58:374-377.

54. Zhang Z, Román GC. Worldwide occurrence of Parkinson's disease: an updated review. Neuroepidemiology 1993;12:195-208.

Manuscrito recibido el 13 de agosto de 2002. Aceptado para publicación, tras revisión, el 19 de marzo de 2003. 


\title{
ABSTRACT National neuroepidemiological study in Colombia (EPINEURO)
}

Objective. To determine the prevalence of eight neurological problems-migraine, cerebrovascular disease, Parkinson's disease, peripheral neuropathy, disorders of neurological development, epilepsy, dementia, and sequelae of head trauma-in

Colombia as a part of the National Neuroepidemiological Study (EPINEURO).

Methods. A cross-sectional survey was carried out in two phases between September 1995 and August 1996. In the first phase persons who might be suffering from a neurological disease were identified, and in the second phase a specific diagnosis was made. A sample of 8910 people were examined, distributed in proportion to the population in each of the five geographical areas of Colombia (central, southwest, northwest, eastern, and Caribbean coast). The evaluation was done using a modified version of the neuroepidemiology protocol of the World Health Organization, along with a simplified physical examination. With the patients with possible dementia a neuropsychological examination designed for this study was used in order to thoroughly examine the cognitive aspects analyzed in the initial mental screening. The scales of Hachinski, Yesavage, and Blessed and the diagnostic criteria of the NINCDSADRDA were used for Alzheimer's disease; the NINDS-AIREN criteria for dementia of vascular origin; and the DSM-IV criteria for all types of dementia. A survey was conducted in order to determine if there were sequelae of head trauma. Persons 12 years old or older were evaluated by an adult neurologist and those under 12 years old by a pediatric neurologist. The prevalences of neurological disorders were calculated by age and sex, for each region and also for the entire country, with $95 \%$ confidence intervals (CIs).

Results. The prevalence per 1000 population for the disorders investigated was: migraine, 71.2 (95\% CI: 65.5 to 76.8); disorders of neurological development, 46.1 (95\% CI: 35.5 to 58.9 ); cerebrovascular disease, 19.9 (95\% CI: 14.3 to 27.4 ); dementia, 13.1 (95\% CI: 8.5 to 19.3); epilepsy, 10.3 (95\% CI: 8.5 to 13.0); peripheral neuropathy, 8.5 (95\% CI: 6.8 to 10.7); sequelae of head trauma, 6.4 (95\% CI: 5.0 to 7.8); and Parkinson's disease, 4.7 (95\% CI: 2.2 to 8.9). In all those cases except head trauma the prevalence was higher among women than among men.

Conclusions. These results make it possible to design more up-to-date health policies aimed at achieving more effective prevention, better control, and more adequate treatment of neurological disorders in Colombia. The easy application of these surveys and the low cost of this type of research make this methodology ideal for countries with shortages of specialized personnel.

\section{Call for Abstracts \\ International Society for Equity in Health Third International Conference on Equity in Health}

The International Society for Equity in Health is seeking abstracts of original research papers to be presented at its Third International Conference, to be held in Durban, South Africa, on June 10-12, 2004. Papers whose abstracts are submitted for presentation should focus on topics such as health determinants, innovative methods for studying and monitoring equity in health; policies, services and interventions that reduce health inequities, and the use of evidence to enhance public accountability and social action geared toward achieving equity in health. There is a special interest in abstracts that reflect a multidisciplinary conceptualization of health equity; that are analytical rather than descriptive; that are not limited to a single measure of health, age group, or population subgroup or whose findings can be extrapolated beyond the particular population studied; and that analyze determinants having an impact on equity outcomes. The deadline for submitting abstracts is 1 December 2003.

\author{
Information: \\ International Society for Equity in Health \\ 256 McCaul Street, Second Foor \\ Toronto, Ontario M5T 1W5-Canada \\ Phone: (416) 978-3763 - Fax: (416) 978-3912 \\ E-mail: leslie.nunez@utoronto.ca \\ Internet: http://www.iseqh.org
}

